\title{
The Environmental Impact on Galaxy Evolution: Highlighting the Structure of the Local Cosmic Web
}

\author{
Antoine Bouchard*, S.-L. Blyth, W. J. G. de Blok, B. W. Holwerda \& \\ K. J. van der Heyden \\ Department of Astronomy, University of Cape Town, Private Bag X3, Rondebosch 7701, \\ Republic of South Africa \\ E-mail: bouchardeast.uct.ac.za
}

\begin{abstract}
Galaxy properties are known to vary across different environments. Higher density environments generally have a higher fraction of early type galaxies than low density ones which are dominated by late types. We present the results of deep, systematic $\mathrm{H}$ I and $\mathrm{H} \alpha$ surveys of galaxies in various environments, namely the Local, Centaurus A, and Sculptor groups. These data are used to perform a quantified analysis of the morphology-density relationship in the Local Universe. In general, the relation is relatively easy to observe, however one population of galaxies clearly depart from the correlation. These objects may be on their first infall into their respective environment. Our analysis allows to extract a wealth of information, not only on galaxy evolution mechanisms and the local $\mathrm{H}$ I mass function but also on the global structure of groups and filaments in the Local Universe. With the advent of SKA pathfinders, targeted, volume-limited, and/or deep blind $\mathrm{H}$ I surveys will allow us to extend our knowledge of the Local structure and its effect on the evolution of galaxies, and highlight the Local Cosmic Web.
\end{abstract}

Panoramic Radio Astronomy: Wide-field 1-2 GHz research on galaxy evolution - PRA2009

June 02 - 052009

Groningen, the Netherlands

${ }^{*}$ Speaker. 


\section{Introduction}

Galaxy properties, especially those of dwarfs that have a shallow gravitational potential well, are affected by a poorly constrained mixture of environmental and internal factors. The neutral hydrogen gas (H I) gas content of these galaxies can easily be disrupted by a plethora of phenomena, including ram pressure from the hot and diffuse Intergalactic Medium (IGM) or winds from massive stars. These gas disruption mechanisms will, in turn, affect the star formation rates of the galaxies and eventually alter their optical morphologies as the stars become older and fainter.

In these proceedings, the global properties of nearby dwarf galaxies are compared to their environment in an attempt to quantify the morphology-density relation. The goal is to assess whether the environment in which galaxies evolve is really the main factor driving their evolution and whether it is possible to determine global environmental properties by studying galactic properties.

\section{Measuring and Understanding the Global Properties of Nearby Dwarf Galaxies}

\subsection{H I Properties}

Several H I surveys have targeted dwarf galaxies in the nearby universe. Figure 1 shows a comparison between the total $\mathrm{H}$ I mass vs absolute $B$-band magnitude for galaxies in the Sculptor group with those found in the Centaurus A group [3,4]. These two groups are fundamentally different environments: Sculptor is a string of loosely bound galaxies [8] while Centaurus A is a denser environment where galaxy interactions are probably much more frequent. In this context, it is not surprising to see that for galaxies fainter then $M_{B}>-13$, there is only a single galaxy with a detected H I mass $M_{H I}<10^{7} M_{\odot}$ in the Centaurus A group while, in Sculptor, the parameter space is populated by a larger number of objects. In fact, there is a dichotomy in the H I properties of Centaurus A galaxies that are fainter than $M_{B}>-13$ : they either contain a very large amount of $\mathrm{H} \mathrm{I}$ $\left(M_{H I}>10^{7} M_{\odot}, M_{H I} / L_{B}>1\right)$ or nothing at all $\left(M_{H I}<10^{6} M_{\odot}\right)$. In this case, the gap in H I properties can probably be explained by the presence of the Centaurus A galaxy itself, a large elliptical galaxy containing an active galactic nucleus (AGN). The jets from the AGN produce very extended lobes [7] that may affect the $\mathrm{H}$ I in surrounding galaxies, e.g., by increasing the intergalactic medium density and the effectiveness of ram pressure stripping or simply by ionizing the gas.

It is interesting to see that the $\mathrm{H}$ I mass function (HIMF) is undetermined for $M_{H I}<10^{7} M_{\odot}$ [10]. Recent attempts to recover this information from existing surveys by using statistical stacking methods have failed to return any results. The CRUMBS (Characterising Radio-Undetected Matter in Baryonic Surveys) project, aims at recovering average H I properties of galaxies that were not detected in NIBLES (Nançay Interstellar Baryon Legacy Extragalactic Survey) [9]. This is done by computing averaged $\mathrm{H}$ I spectra by coadding all $\mathrm{H}$ I non-detections. Figure 2 shows a breakdown of $\mathrm{H}$ I masses with respect to absolute B-band magnitude [1]; H I properties are completely unconstrained for galaxies fainter than $M_{B}>-13$ or $M_{H I}<10^{7} M_{\odot}$.

Currently, the number of objects with detected H i masses smaller $10^{7} M_{\odot}$ is simply too low to draw any statistically significant conclusion. To constrain the shape of the faint end of the HIMF and reasonably assess whether or not it is dominated by environmental properties, we will require a large scale (volume limited) observing program that may not be possible to undertake with the currently existing facilities. 


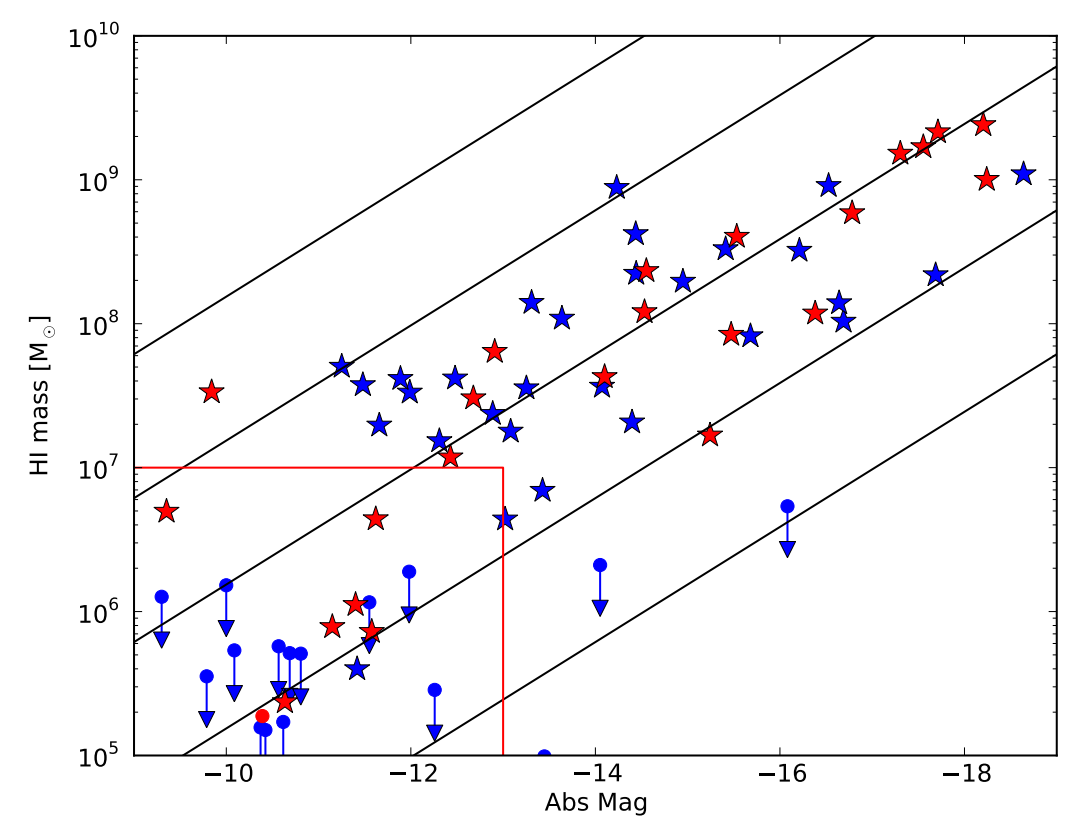

Figure 1: H I mass vs. absolute blue magnitude for galaxies of the Sculptor group (red) and of the CenA group (blue). Star shaped markers are used to show actual H I detections while circles are upper limits for undetected galaxies. Lines of constant $\mathrm{H}$ I mass to light ratios are overplotted in black.

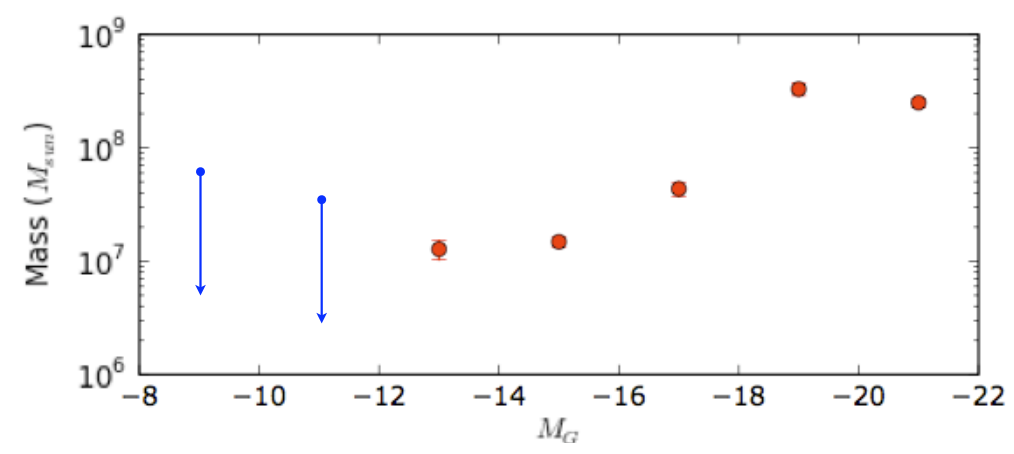

Figure 2: Average $\mathrm{H}$ I mass vs. absolute $\mathrm{G}$ magnitude for galaxies that were not detected in $\mathrm{H}$ I. These are results form the CRUMBS project, an H I stacking experiment [1,9]. 


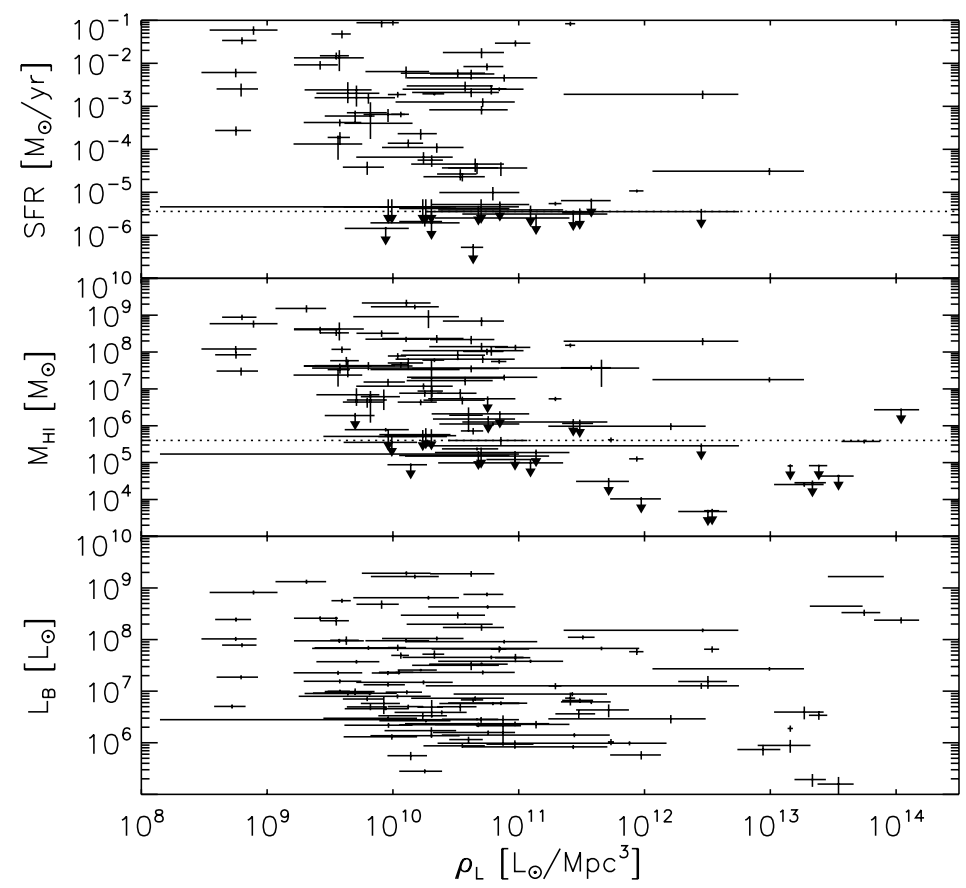

Figure 3: Star formation rate, $\mathrm{H}$ I mass and $B$-band luminosity as a function of environmental density for galaxies in the Local, Sculptor and Centaurus A groups. Galaxies in red are objects that are deviating from the 'expected' morphology-density relation. See text for details.

\subsection{Star Formation Rates}

If global galaxy properties are indeed a strong function of their environment, there must exist a way to quantify the relationship. Figure 3 shows the star formation rate (SFR), $M_{H I}$ and $B$-band luminosity $\left(L_{B}\right)$ as a function of the instantaneous 3-D galaxy distribution $\left(\rho_{L}\right)$, see [2] for more details. There is a statistically significant correlation between the first two parameters (SFR and $M_{H I}$ ) and environmental density although the number of outliers makes the correlations difficult to observe. Furthermore, the correlations seem to completely disappear under some circumstances [6]: more investigations are required to properly assess the role of environment on galaxy evolution.

Possibly, one of the reason for the large scatter in the correlations is that environmental properties are not properly described by $\rho_{L}$ but that orbital parameters also need to be taken into account. For instance, some of these objects might be on their first infall onto their respective structures: the environment has not yet started to influence their properties. Other physical processes may also be at work in these galaxy groups. The prospect of inhomogeneous IGM and/or IGM motion (similar to winds) should also be considered as it would affect galaxies in ways that we cannot currently quantify.

High resolution and high sensitivity $\mathrm{HI}$ imaging of a large number of galaxies could help resolve these issues. Ram pressure, tidal stripping, or other environmental mechanisms often affect the outer $\mathrm{H}$ I envelopes of galaxies. 


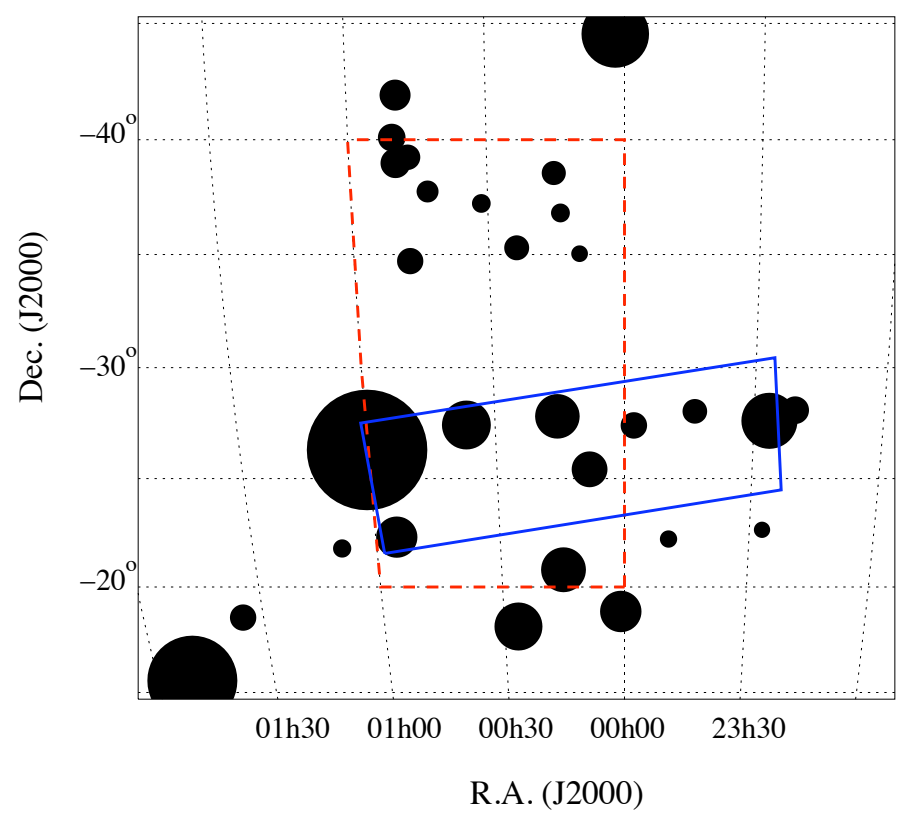

Figure 4: Two possible regions for a Local Cosmic Web survey in the Sculptor region. Circles show the position of nearby galaxies, the size of the symbols are inversely proportional to the distance.

\section{Searching for the Local Cosmic Web}

To distinguish between environmental and internal drivers for galaxy properties evolution, one needs to observe a complete sample of galaxies inside a specific volume. The Karoo Array Telescope (MeerKAT) will be the ideal instrument for this kind of study.

A systematic, targeted H I line survey of all galaxies in the Local Volume $(D<20 \mathrm{Mpc})$ with MeerKAT would define both the faint-end of the HIMF and its environmental dependence, as well as the internal properties that may be affected by environment (e.g., by ram pressure). Within 3 hours of integration time per galaxy, we will reach a $5 \sigma$ detection limit of $8 \times 10^{6}(D / 20 \mathrm{Mpc})^{2} M_{\odot}$ (assuming an unresolved source with $20 \mathrm{~km} \mathrm{~s}^{-1}$ velocity dispersion, this may imply smoothing or rebinning of the data), thereby increasing the number of $\mathrm{H}$ I detections in the currently undefined end of the HIMF.

By using different data reduction parameters, MeerKAT will simultaneously provide different sets of images with sensitivity to low column density H I $\left(\mathrm{N}_{H I}=10^{19} \mathrm{~cm}^{-2}\right.$ in 3 hours) and with high spatial resolution ( $\sim 8^{\prime \prime}$ resolution). This will be ideal to study both galaxy interactions and internal processes, hence allowing us to fully disentangle the effects of different processes on the evolution of dwarf galaxies.

To complement the targeted, volume-limited survey, a deep blind survey of a strip of sky, tentatively located in the Sculptor group region (Figure 4), will allow direct observation of the Local Cosmic Web. A $5 \sigma$ detection limit of $\mathrm{N}_{H I}=10^{18} \mathrm{~cm}^{-2}$ with $90^{\prime \prime}$ resolution can be reached in 155 hours. This is the level at which [5] saw the first hints of $\mathrm{H}$ I bridges between galaxies. 


\section{Conclusion}

The HIMF remains undefined for H I mass values smaller than $10^{7} M_{\odot}$. This is despite several observational attempts at constraining it. As shown by the pronounced differences between the Centaurus A and Sculptor observations, there could be physical reasons why the faint end of this function remains elusive and why its shape may have strong environmental dependencies below this limit.

The effect of environment may be more complicated than a simple galaxy density measure possibly represents. Cold gas accretion, inhomogeneous IGM or even motion in the IGM may also impact dwarf galaxy evolution and the only way to determine the role these phenomena play resides in future large scale observational projects.

\section{References}

[1] Blyth, S. L., Bouchard, A., van der Heyden, K., de Blok, W. J. G., van Driel, W., Kraan-Korteweg, R., \& Ramatsoku, M. 2009, this conference

[2] Bouchard, A., Costa, G. S. D., \& Jerjen, H. 2009, AJ, 137, 3038

[3] Bouchard, A., Jerjen, H., Da Costa, G. S., \& Ott, J. 2005, AJ, 130, 2058

[4] -. 2007, AJ, 133, 261

[5] Braun, R. 2004, New Astronomy Review, 48, 1271

[6] Côté, S., Draginda, A., Skillman, E. D., \& Miller, B. W. 2009, AJ, 138, 1037

[7] Hardcastle, M. J., Cheung, C. C., Feain, I. J., \& Stawarz, Ł. 2009, MNRAS, 393, 1041

[8] Jerjen, H., Freeman, K. C., \& Binggeli, B. 1998, AJ, 116, 2873

[9] van Driel, W. 2009, in proceedings PRA2009

[10] Zwaan, M. A., Meyer, M. J., Staveley-Smith, L., \& Webster, R. L. 2005, MNRAS, 359, L30 